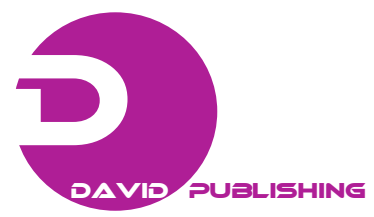

\title{
Analytical Survey on Aflatoxin B1, Ochratoxin A, Fumonisin B1 and Fumonisin B2 of Cambodian Rice
}

\author{
Dalin Ly* ${ }^{1}$, Sarom Men ${ }^{1}$, Vibol San ${ }^{2}$, Noel Durand ${ }^{3}$, Jean-Claude Manez ${ }^{3}$ and Didier Montet ${ }^{3}$ \\ 1. Royal University of Agriculture, P.O. Box 2696, Phnom Penh, Cambodia \\ 2. Royal University of Phnom Penh, Russian Federation Blvd. Tek Laak 2 Commune, P.O. Box 12157, Phnom Penh, Cambodia \\ 3. UMR Qualisud, Centre de Coopération Internationale en Recherche Agronomique pour le Développement, CIRAD, 34398
}

Montpellier Cedex 5, France

*Corresponding author's e-mail: dalinely@rua.edu.kh

\begin{abstract}
An analytical survey of mycotoxins was carried out by collecting 40 paddy rice samples during harvest and storage stage from two large rice producing provinces namely Prey Veng and Takeo of Cambodia. On the other hand, 20 milled rice samples collected from four markets (Kandal, Oreusey, Beungkengkang and Chbar-ampov) in Phnom Penh were also studied. Moisture contents were measured and samples were dried until moisture content raised to $13 \%$, after that the samples were packed in poly-ethylene plastic bag before exporting to CIRAD in France to analyze for aflatoxin B1, total aflatoxins, ochratoxin A, fumonisin B1 and fumonisin B2 using immunoaffinity column and HPLC. These toxins were extracted using a mixture of $80 \%$ methanol and $20 \%$ water, then the extracts were purified and the quantification was done by HPLC with fluorescent detection column. The analytical results revealed that one sample (1.67\%) out of 60 rice samples was contaminated by fumonisin B1 and B2 at $0.8 \mu \mathrm{g} / \mathrm{kg}$ and $0.1 \mu \mathrm{g} / \mathrm{kg}$, respectively. One milled rice sample was contaminated with aflatoxin B1 and total aflatoxins with the same levels of $0.75 \mu \mathrm{g} / \mathrm{kg}$. Contamination levels of aflatoxin B1, total aflatoxins, fumonisin B1 and B2 were found in some samples, but the amounts of contaminations were below the European Union detection level (regulation No.1881/2006). Therefore, this study results demonstrated that Cambodia rice (paddy and milled rice) was in good quality and was safe for consumption in local and/or international consumers.
\end{abstract}

Key words: Analytical survey, aflatoxin B1, ochratoxin A, fumonisin B1 and B2, Cambodian Rice. 